\title{
BIOESTIMULANTE NA PRODUÇÃO DE MUDAS DE VIDEIRA CV. CRIMSON SEEDLESS
}

\author{
Biostimulant on seedling production of grape cv. Crimson Seedless
}

Rafael Ferreira Ribeiro ${ }^{1}$; Jackson Teixeira Lobo ${ }^{2 *}$; Ítalo Herbert Lucena Cavalcante ${ }^{3}$; Igor Gomes Pouso Tenreiro ${ }^{4}$;

Deisiele Diniz Lima ${ }^{4}$

\author{
${ }^{1}$ Engenheiro Agrônomo; Global Crops; rafaelribeiro87@gmail.com \\ ${ }^{2}$ Mestrando; Programa de Pós-Graduação em Produção Vegetal; Universidade Federal do Vale do São Francisco; \\ jackson_lob@hotmail.com \\ (*Autor para correspondência) \\ ${ }^{3}$ Professor Doutor; Universidade Federal do Vale do São Francisco; italo.cavalcante@univasf.edu.br \\ ${ }^{4}$ Engenheiro Agrônomo; Universidade Federal do Vale do São Francisco; igortenreiro@gmail.com; deisiele_diniz@hotmail.com
}

Artigo enviado em 26/02/2017, aceito em 01/09/2017 e publicado em 20/12/2017.

Resumo - A produção de mudas com qualidade é fundamental na exploração das culturas agrícolas, e os bioestimulantes apresentam-se como ferramentas benéficas nessa fase da planta. O estudo objetivou avaliar as diferentes concentrações de bioestimulante composto por extrato de algas, no favorecimento da expansão radicular e desenvolvimento vegetativo inicial de mudas de uva, cultivar Crimson Seedless enxertadas em porta-enxerto SO4. O experimento foi realizado em viveiro comercial na cidade de Petrolina-PE. O delineamento experimental utilizado foi o inteiramente casualizado, com cinco tratamentos e quatro repetições. Os tratamentos foram: (T1) 0; (T2) 0,75; (T3) 1,12; (T4) 1,5 e (T5) $1,87 \mathrm{~mL}$ planta $^{-1}$ do bioestimulante. Aos 45 dias após a enxertia foram analisados o comprimento e diâmetro da brotação do enxerto; área foliar; diâmetro, densidade, área, comprimento e matéria seca das raízes e matéria seca da parte aérea. Os dados foram submetidos à análise de variância e análise de regressão polinomial. Os melhores resultados foram proporcionados pela aplicação da dose $0,75 \mathrm{~mL}$ planta- ${ }^{-1} \mathrm{O}$ aumento da dose causou redução na expressão das variáveis. Conclui-se que o bioestimulante é uma ferramenta importante na produção de mudas com qualidade.

Palavras-Chave - Vitis vinifera, Ascophyllum nodosum, propagação vegetativa, reguladores vegetais.

\begin{abstract}
The seedling production with quality is essential for growing agricultural crops, and the biostimulants are beneficial tools for this plant phase. The study aimed to evaluate the different concentrations of biostimulant containing algae extract on root expansion and initial vegetative development of grape seedlings cv. Crimson Seedless grafted on SO4 rootstock. The experiment was conducted in commercial nursery in Petrolina county, Pernambuco state, Brazil. The experimental design used was the completely randomized design, with five treatments and four replicates. The treatments were: (T1) 0; (T2) 0.75; (T3) 1.12; (T4) 1.5 and (T5) $1.87 \mathrm{~mL}$ plant $^{-1}$ of the biostimulant. At 45 days after grafting the length and diameter of the graft sprouting; leaf area; diameter, density, area, length, and dry weight of roots and shoots were analyzed. The data were subjected to analysis of variance and polynomial regression. The best results were provided by $0.75 \mathrm{~mL}_{\text {plant }}{ }^{-1}$ biostimulant dose. Increasing biostimulant doses caused the reduction of variables expression. It is concluded that the biostimulant is an important tool for production of quality grape seedlings.
\end{abstract}

Keywords - Vitis vinifera, Ascophyllum nodosum, vegetative propagation, plant regulators. 


\section{INTRODUÇÃO}

No vale do Submédio São Franscisco, a viticultura exerce importante papel econômico e tem expandido devido a fatores climáticos favoráveis e uso da irrigação que possibilita sua produção durante todo o ano (SOUZA et al., 2016). De acordo com Pinillos et al. (2016) os mercados de uva de mesa, principalmente o mercado externo é exigente quanto a qualidade dos frutos, e nesse contexto a cultivar apirênica Crimson Seedless tem sido bastante explorada nas condições tropicais, pelas suas qualidades de vigor, produtividade, tamanho de baga e cor atraente (LEÃO; SOARES; RODRIGUES, 2009).

Com a intensificação da exploração vitícola, há uma crescente demanda por mudas com elevado desempenho agronômico, seja para compor novos parreirais ou em substituição aos existentes.

Uma importante forma de reprodução da videira é através da propagação vegetativa. O processo de estaquia combinado com enxertia garante que as novas plantas mantenham características genotípicas da planta-mãe, assim como um bom desenvolvimento radicular, dando condições à variedade enxertada de se estabelecer e expressar seu potencial genético (ALBUQUERQUE; RODRIGUES; ALBUQUERQUE-NETO, 2008).

Objetivando um melhor e mais rápido desenvolvimento das mudas, novos insumos têm sido empregados no processo produtivo, destacando-se a utilização dos bioestimulantes. Os bioestimulantes incluem substâncias e microorganismos com capacidade para alterar o metabolismo vegetal favorecendo seu desenvolvimento (CALVO; NELSON; KLOEPPER, 2014). Aplicados diretamente sobre a planta ou na rizosfera, os bioestimulantes podem contribuir na melhoria das propriedades físicoquímicas do solo, na absorção, translocação e uso dos nutrientes pelas plantas, incluindo aumento de resistência a estresses abióticos, podendo ser usado nas diversas fases do desenvolvimento vegetal, desde a germinação até a maturidade (CALVO; NELSON; KLOEPPER, 2014; DU JARDIN, 2015).

Dentre as substâncias naturais às quais se atribuem efeitos bioestimulantes, a utilização de extrato de algas na agricultura vem sendo descrita em diversas pesquisas (KHAN et al. 2009; CRAIGIE, 2011; ARIOLI; MATTNER; WINBERG, 2015).

Os extratos de alga possuem em sua composição macro e micronutrientes, aminoácidos e hormônios vegetais; a alga Ascophyllum nodosum em especial possui auxinas, citocininas, giberelinas e ácidos abscísicos, contendo também compostos capazes de estimular a produção desses hormônios pelas plantas (KHAN et al., 2009; MACKINNON et al., 2010; SHARMA et al., 2012).

A aplicação de produtos à base do extrato da alga Ascophyllum nodosum tem proporcionado resultados positivos no aumento da produtividade em tomate (KOYAMA et al., 2012), produção e qualidade de uva sob condição de solo alcalino (SABIR et al., 2014), indução de resistência ao déficit hídrico em soja (MARTYNENKO et al., 2016) e laranja (SPANN; LITTLE, 2011), e resistência ao ataque de patógenos em pepino (JAYARAMAN; NORRIE; PUNJA, 2011). No entanto, novas pesquisas são necessárias considerando que as respostas das plantas variam em função da espécie, concentração do extrato e das condições ambientais.

Neste sentido, objetivou-se com esse estudo, avaliar as diferentes concentrações do bioestimulante composto por extrato de algas, no favorecimento da expansão radicular e desenvolvimento vegetativo inicial de mudas de videira, cultivar Crimson Seedless enxertadas em estacas do porta-enxerto SO4.

\section{MATERIAL E MÉTODOS}

O experimento foi conduzido durante os meses de dezembro de 2015 e janeiro de 2016, em viveiro comercial especializado na produção de mudas de videiras, na cidade de Petrolina-PE. O viveiro foi coberto com tela de sombreamento $50 \%$ e a irrigação foi realizada por micro aspersão invertida de vazão $45 \mathrm{~L} \mathrm{~h}^{-1}$, acionada periodicamente três vezes ao dia durante 15 minutos, a umidade relativa do ar média registrada durante o experimento foi de $80 \%$ e a temperatura do ar de $23^{\circ} \mathrm{C}$.

O delineamento experimental foi inteiramente casualizado com cinco tratamentos e quatro repetições e dez mudas por parcela, totalizando duzentas mudas. Os tratamentos foram doses de bioestimulante: $0 ; 0,75$; 
1,$12 ; 1,5$ e $1,87 \mathrm{~mL}$ planta $^{-1}$, equivalentes respectivamente a $0 ; 0,08 ; 0,11 ; 0,15 ; 0,19 \mathrm{~mL}_{\text {planta }}{ }^{-1}$ do extrato de alga. $\mathrm{O}$ produto foi diluído em água nas seguintes dosagens $0 ; 2,5 ; 3,7 ; 5,0$ e $6,2 \mathrm{~mL} \mathrm{~L}^{-1}$. O bioestimulante usado foi o Solofull (Global Crops Agri Solutions $\left.{ }^{\circledR}\right)$, produto à base da alga Ascopbyllum nodosum (L.) Le Jolis, composto por $4 \%(\mathrm{p} / \mathrm{p})$ de $\mathrm{K}_{2} \mathrm{O}$, $6 \%$ (p/p) de Carbono Orgânico Total, 10,0\% de extrato de algas e 0,25\% de ácido cítrico.

A variedade copa apirênica estudada foi a Crimson Seedless enxertada pelo método de fenda cheia no porta-enxerto SO4 com $30 \mathrm{~cm}$ de comprimento. As estacas foram plantadas após $24 \mathrm{~h}$ de imersão do produto comercial Radimax ${ }^{\circledR}\left(2,25 \mathrm{~g} \mathrm{~L}^{-1} \mathrm{de}\right.$ ácido indolbutírico) na proporção $1,5 \mathrm{ml}$ planta $^{-1} \mathrm{em}$ sacos de polietileno com volume de $250 \mathrm{~cm}^{3}$. O substrato utilizado foi uma mistura homogênea de terra vegetal com areia grossa, na proporção 3:1.

A aplicação dos tratamentos foi feita via solo em uma única vez, logo após o plantio. As análises foram realizadas 45 dias após inicio do experimento, as mudas foram conduzidas ao Laboratório de Fruticultura do Curso de Engenharia Agronômica da Universidade Federal do Vale do São Francisco UNIVASF, onde foram determinados comprimento de brotação do enxerto $(\mathrm{cm})$, diâmetro da brotação $(\mathrm{mm})$, área foliar $\left(\mathrm{cm}^{2}\right)$, diâmetro de raízes $(\mathrm{mm})$, densidade de raízes $\left(\mathrm{mm} . \mathrm{mm}^{-2}\right)$, área de raízes $\left(\mathrm{mm}^{2}\right)$, comprimento de raízes $(\mathrm{cm})$, matéria seca de raízes $(\mathrm{mg})$ e matéria seca da parte aérea (g).

As análises de comprimento e diâmetro da brotação do enxerto foram realizadas nas mudas intactas com auxílio de fita milimetrada e paquímetro, para a análise de área foliar as folhas foram destacadas, lavadas, secas e escaneadas, a determinação quantitativa foi realizada pelo software ImageJ v. 1.48. Para as análises de diâmetro, densidade, área e comprimento de raízes, o sistema radicular foi lavado em água corrente, logo após, separado e tratado com solução de azul de metileno $(0,5 \%)$ para digitalização em bandeja de vidro com água, as imagens foram geradas em formato TIFF 5.0 e processadas no software "Delta-T SCAN®", seguindo as recomendações de Kirchhof e Pendar (1993). Para mensuração da matéria seca das raízes e matéria seca da parte aérea, as respectivas partes foram secas em estufa de ventilação forçada à $60^{\circ} \mathrm{C}$ por 72 horas e pesadas posteriormente em balança de precisão.

Os dados obtidos foram submetidos à análise de variância (ANOVA), e análise de regressão polinomial a $5 \%$ de significância no software SigmaPlot ${ }^{\circledR}$, considerando significativo $\mathrm{P} \leq 0,05$.

\section{RESULTADOS E DISCUSSÃO}

De acordo com o resultado da análise de variância, pode-se afirmar que o uso do bioestimulante à base do extrato de algas promoveu um aumento significativo para as variáveis: comprimento da brotação do enxerto, densidade de raízes, área de raízes, comprimento de raízes, matéria seca de raízes e matéria seca da parte aérea. Os resultados para área foliar, matéria seca da parte aérea e comprimento da brotação do enxerto estão ilustrados na figura 1 .

Para a área foliar (Figura 1A), todas as doses do bioestimulante proporcionaram aumento em relação ao controle, especialmente $0,75 \mathrm{~mL}$ planta $^{-1}$, AbdelMawgoud et al. (2010) também observaram um aumento significativo no tamanho das folhas de melancieira tratadas com extrato de algas marinhas. De acordo com Wally et al. 2013, as algas marrons estimulam a produção de citocininas, fitormônio responsável pela divisão celular e consequentemente pela expansão foliar (SPAEPEN; VANDERLEYDEN; OKON, 2009).

O diâmetro da brotação do caule não demonstrou diferença significativa para as doses de bioestimulante testadas sendo o valor médio da variável 3,23 $\mathrm{mm}$. Esse fato pode estar associado ao curto período de tempo entre o início do experimento e o período de avaliação. Dantas et al. (2012) também não identificaram efeito significativo para o uso de bioestimulante para a variável diâmetro do caule em mudas de tamarindo.

Verificou-se que a matéria seca da parte aérea e o comprimento de brotação do enxerto foram influenciados pelas doses de bioestimulantes (Figura 1B e 1C). Para ambas as variáveis, a distribuição dos dados foi semelhante, apresentando superioridade dos tratamentos com as doses 0,75; 1,12 e 1,5 mL planta $^{-1}$ em relação à testemunha, da mesma forma como ocorreu para a variável área foliar os melhores 
resultados foram para a dose $0,75 \mathrm{~mL}$ planta $^{-1}$ do bioestimulante.
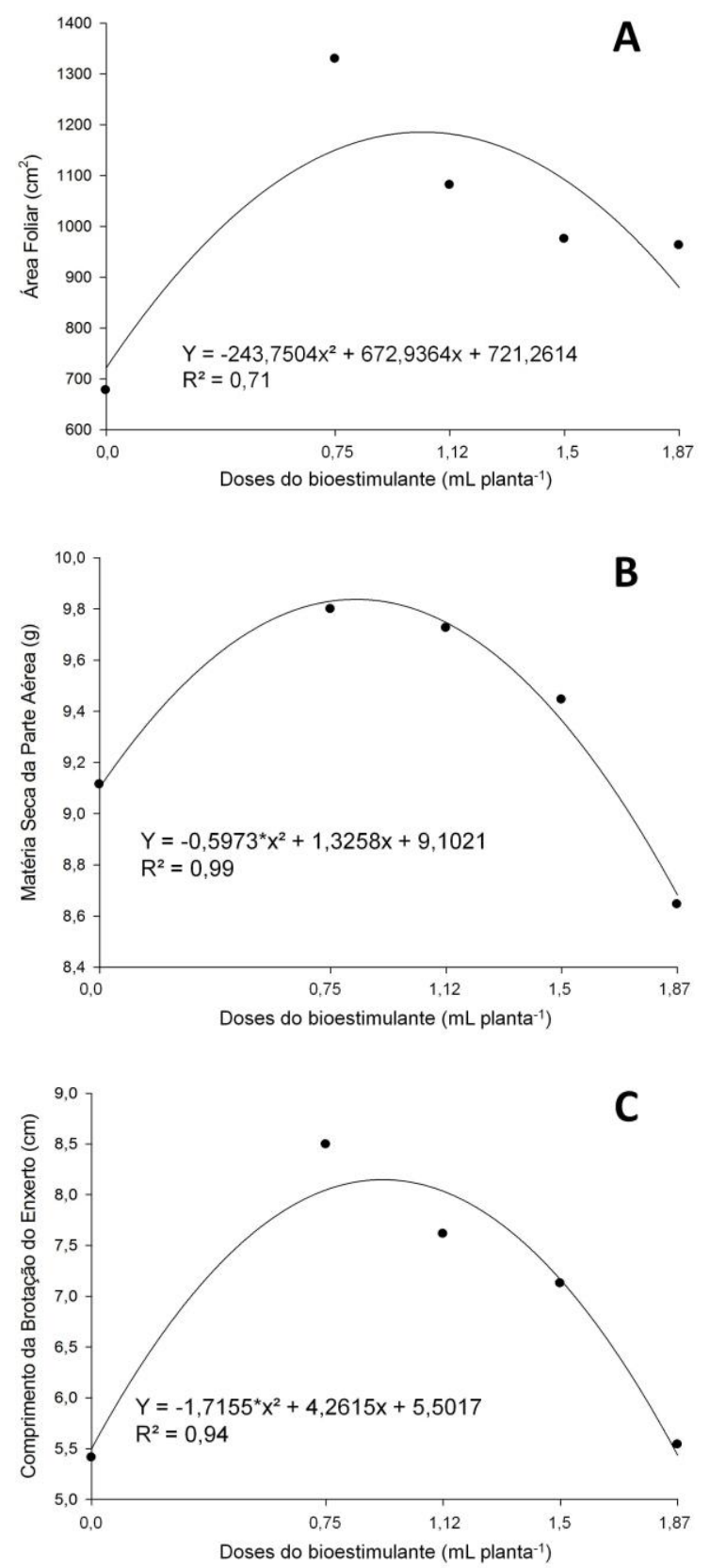

Figura 1. Efeito das diferentes doses de bioestimulante na parte aérea: Área foliar (A); Matéria seca da parte aérea (B) e Comprimento de brotação do enxerto (C), de mudas de videira cv. Crimson Seedless enxertadas em porta-enxerto SO4.
Verifica-se que a maior dose estudada promoveu diminuição em relação às doses intermediárias independentemente da variável estudada, além de clorose nas folhas e engrossamento das raízes. Para a matéria seca da parte aérea, a máxima dose estimada foi de $0,83 \mathrm{~mL}$ planta $^{-1}$ proporcionando o seu maior incremento, e para o comprimento de brotação do enxerto o valor pra sua máxima expressão seria de $0,93 \mathrm{~mL}$ planta $^{-1}$.

O comportamento similar das doses entre as variáveis analisadas mostrou que o bioestimulante utilizado em dose adequada promove alterações positivas no metabolismo e fisiologia das plantas, melhorando uniformemente suas características fitotécnicas.

Silva et al. (2016) observaram em mudas de Annona glabra, que para o número de folhas e matéria seca da parte aérea os melhores resultados foram obtidos com a dose de $2,0 \mathrm{~mL} \mathrm{~L}^{-1} \mathrm{H}_{2} \mathrm{O}$ do extrato de Ascophyllum nodosum, doses maiores apresentaram redução na expressão das variáveis. Os autores associam a redução das características fitotécnicas à condição de estresse salino causada pelas doses superiores.

Em mudas de mamoeiro, a dose ideal para a matéria seca da parte aérea verificada por Guimarães et al. (2015) foi de 5,97 $\mathrm{mL} \mathrm{L}^{-1}$ do bioestimulante Root ${ }^{\circledR}$, apresentando resultados decrescentes para taxa maior. Embora a dose ideal para mudas de mamão tenha sido maior que a verificada para mudas de uva, o comportamento dos dados foi semelhante, aumentando até a ideal e reduzindo em seguida.

Em relação às variáveis do sistema radicular, não houve diferença estatística para o diâmetro de raízes sendo o valor médio observado de 1,47 mm. Houve incremento significativo para a variável densidade de raízes em função dos tratamentos, sendo a melhor dose a de $0,75 \mathrm{~mL}$ planta $^{-1}$, com média de $0,0322 \mathrm{~mm} \cdot \mathrm{mm}^{-2}$, valor $57 \%$ superior ao da testemunha, apresentando dose máxima de 0,67 mL planta $^{-1}$ a partir da análises de regressão (Figura 2A). De acordo com Fagan et al. (2015) há uma relação favorável Auxina/Citocinina no uso do bioestimulante até uma determinada dose, acima desta, a relação diminui e prevalece o efeito citocínico. 

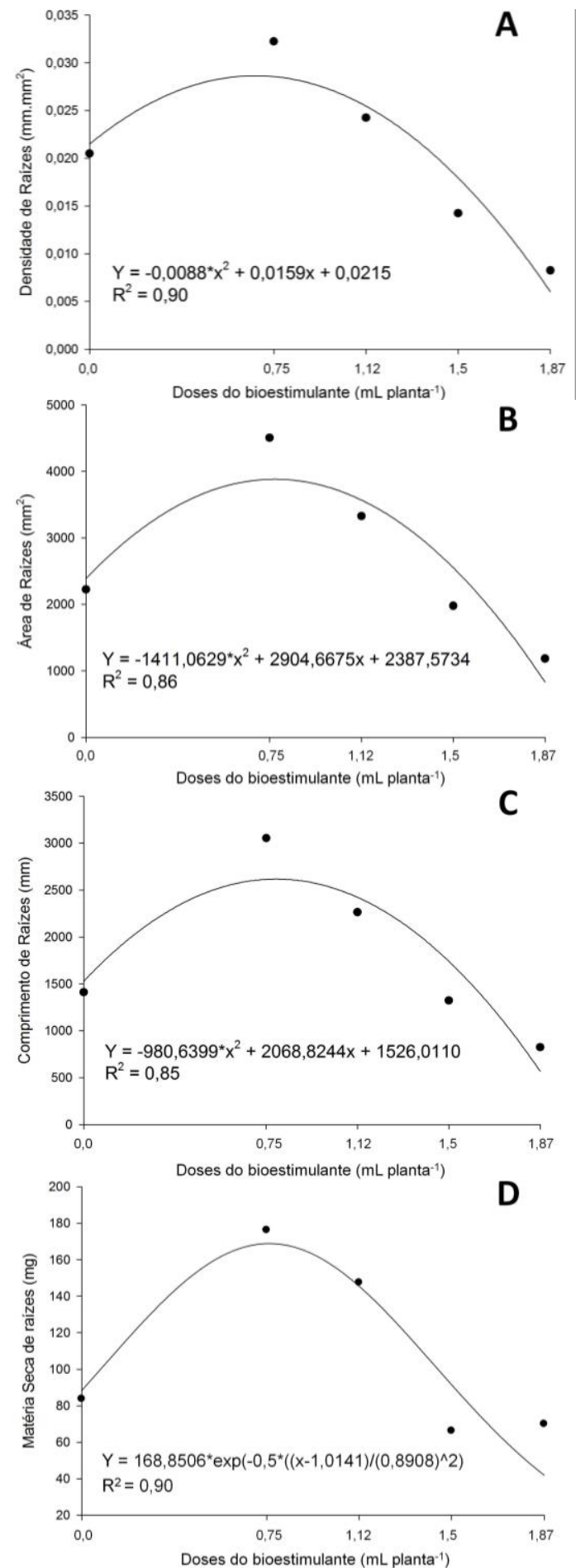

Figura 2. Efeito das diferentes doses de bioestimulantes no sistema radicular: Densidade de raízes (A); Área de raízes (B); Comprimento de raízes (C) e Matéria Seca de raízes (D), de mudas de videira cv. Crimson Seedless enxertada em porta-enxerto $\mathrm{SO} 4$.
As médias superiores para as variáveis área da raiz e comprimento foram verificadas nas doses $0,75 \mathrm{e}$ 1,12 $\mathrm{mL}$ planta $^{-1}$, com valores de área de 4505,88 $\mathrm{mm}^{2}$ e $3328,24 \mathrm{~mm}^{2}$, respectivamente, apresentando uma dose máxima próxima de $0,73 \mathrm{~mL}$ planta $^{-1}$ e valores de comprimento de 3052,82 $\mathrm{mm}$ e $2263,98 \mathrm{~mm}$, com dose máxima estimada pelo teste de regressão de $0,71 \mathrm{~mL}$ planta $^{-1}$ (Figura 2B e 2C). Para a maior dose testada $\left(1,87 \mathrm{~mL}\right.$ planta $\left.^{-1}\right)$ houve uma redução no comprimento de raiz, em aproximadamente $73 \%$ comparado ao valor obtido pelo tratamento dois $\left(0,75 \mathrm{~mL}\right.$ planta $\left.^{-1}\right)$.

De acordo com Kumar e Sahoo (2011) algas e extratos de algas marinhas atuam nas propriedades do solo como condicionadores, aumentando a sua retenção de umidade e favorecendo o crescimento de microrganismos neste. Características estas que podem ter contribuído para o aumento da área radicular.

Em relação à matéria seca das raízes, as doses 0,75 e $1,12 \mathrm{~mL}$ planta $^{-1}$ apresentaram valores similares entre si, e superiores aos demais tratamentos, com médias de 176,25 mg e 147,50 mg respectivamente, de acordo com o teste de regressão a dose máxima encontrada foi de $0,74 \mathrm{~mL}$ planta $^{-1}$ (Figura 2D), mostrando assim que a dose testada de $0,75 \mathrm{~mL}$ planta1 proporciona um desenvolvimento favorável para o enraizamento das estacas considerando a maioria dos parâmetros analisados nesse estudo. Os resultados para incremento no comprimento e matéria seca de raiz encontrados diferem dos encontrados por Bezerra et al. (2007), que estudando os efeitos de bioestimulantes na produção de mudas de alface, verificaram que o aumento nas concentrações dos produtos proporcionou um acréscimo no comprimento e matéria seca da raiz, de forma linear.

Entretanto, Silva et al. (2012) avaliando o uso de diferentes bioestimulantes aplicados em mudas de videira Thompson Seedless enxertada sobre portaenxertos 'SO4', 'Paulsen 1103', 'IAC 572' e 'Harmony', não encontraram efeito positivo sobre a variável matéria seca de raízes, de acordo com os autores o resultado pode estar relacionado ao aumento da concentração salina no ambiente radicular pelas doses utilizadas, impedindo o efeito benéfico dos bioestimulantes; de forma comparativa Albuquerque, Rodrigues e Albuquerque-Neto (2008) não 
identificaram diferença para enraizamento de estacas de videira tratadas com diferentes doses de bioestimulantes e ácido indolbutírico, indicando que as respostas variam em função de outros fatores além dos estudados.

De modo geral, no presente estudo a aplicação da dose $0,75 \mathrm{~mL}$ planta $^{-1}$ apresentou melhor desempenho, e conforme o aumento de dose constatou-se redução na expressão fisiológica para as diferentes variáveis. Resultados semelhantes foram encontrados em experimentos com amora-preta e feijão-da-china (KAVIPRIYA et al., 2011; DIAS et al., 2012), em que ao testar diferentes doses de bioestimulantes, foi identificado a existência de uma dose máxima para ganho de crescimento das plantas e que doses superiores a esta causaram prejuízo ao desenvolvimento das espécies.

\section{CONCLUSÕES}

Há influência positiva do bioestimulante à base de extrato de alga Ascophyllum nodosum na produção de mudas de videira cr. Crimson Seedless enxertadas sobre porta-enxerto SO4, sendo a dose de 0,75 $\mathrm{mL}$ planta $^{-1}$ a mais recomendada. Em doses superiores o bioestimulante causa depressão das características fitotécnicas.

\section{REFERÊNCIAS}

ABDEL-MAWGOUD, A.M.R.; TANTAWAY, A.S.; HAFEZ, M.M.; HABIB, H.A.M. Seaweed Extract Improves Growth, Yield and Quality of Different Watermelon Hybrids. Research Journal of Agriculture and Biological Sciences, v. 6, n. 2, p. 161-168, 2010.

ALBUQUERQUE, T.C.S.; RODRIGUES, F.M.; ALBUQUERQUE-NETO, A.A.R. Efeito de bioestimulantes na brotação e enraizamento de estacas do porta-enxerto SO4 (Vitis Berlandieri $x$ Vitis Riparia). In: CONGRESSO BRASILEIRO DE FRUTICULTURA, 20, 2008, Vitória, ES. Anais...Vitória: SBF, 2008.

ARIOLI, T.; MATTNER, S.W.; WINBERG, P.C. Applications of seaweed extracts in Australian agriculture: past, present and future. Journal of Applied Phycology, v. 27, p. 2007-2015, 2015.
BEZERRA, P.S.G.; GRANJEIRO, L.C.; NEGREIROS, M.Z.; MEDEIROS, J.F. Utilização de bioestimulante na produção de mudas de alface. Cientifica, v. 35, n. 1, p. 46-50, 2007.

CALVO, P.; NELSON, L.; KLOEPPER, J.W. Agricultural uses of plant biostimulants. Plant Soil, v. 383, p. 3-41, 2014.

CRAIGIE, J.S. Seaweed extract stimuli in plant science and agriculture. Journal of Applied Phycology, v. 23, p. 371 393, 2011.

DANTAS, A.C.V.L.; QUEIROZ, J.M.O.; VIEIRA, E.L.; ALMEIDA V.O. Effect of gibberellic acid and the biostimulant stimulate $\AA$ on the initial growth of tamarind. Revista Brasileira de Fruticultura, v. 34, n. 1, p. 08-14, 2012.

DIAS, J.P.T.; TAKAHASHI, K.; DUARTE-FILHO, J.; ONO, E. O. Bioestimulante na promoção da brotação em estacas de raízes de amoreira-preta. Revista Brasileira de Fruticultura, v. 34, n. 1, p. 01-07, 2012.

DU JARDIN, P. Plant biostimulants: Definition, concept, main categories and regulation. Scientia Horticulturae, v. 196, p. 3-14, 2015.

FAGAN, E.B.; ONO, E.O.; RODRIGUES, J.D.; CHALFUN JÚNIOR, A.; DOURADO NETO, D. Fisiologia Vegetal: Reguladores Vegetais. São Paulo, SP: Andrei Editora LTDA, 2015.

GUIMARÃES, I.P.; PAIVA, E.P.; ALMEIDA, J.P.N.; ARRAIS, I. G.; CARDOSO, E. A.; SÁ, F.V.S. Produção de mudas de três acessos de mamoeiro sob doses do bioestimulante Root ${ }^{\circledR}$. Revista de Ciências Agrárias, v. 38, n. 3, p. 414-421, 2015.

JAYARAMAN, J.; NORRIE, J.; PUNJA, Z.K. Commercial extract from the brown seaweed Ascophyllum nodosum reduces fungal diseases in greenhouse cucumber. Journal of Applied Phycology, v. 23, p. 353-361, 2011.

KAVIPRIYA， R.; DHANALAKSHMI， P.K.; JAYASHREE, S.; THANGARAJU, N. Seaweed extract as a biostimulant for legume crop, green gram. Journal of Ecobiotechnology, v. 3, n. 8, p. 16-19, 2011.

KHAN, W.; RAYIRATH, U.P.; SUBRAMANIAN, S.; JITHESH, M.N.; RAYORATH, P.; HODGES, D.M.; CRITCHLEY, A.T.; CRAIGIE, J.S.; NORRIE, J.; PRITHIVIRAJ, B. Seaweed Extracts as Biostimulants of Plant Growth and Development. Journal of Plant Growth Regulation, v. 28, p. 386-399, 2009. 
KIRCHHOF, G.; PENDAR, K. Delta-T SCAN User Manual. Cambridge: Delta-T Scan Devices Ltd, 1993, 244p.

KOYAMA, R.; BETTONI, M.M.; RODER, C.; ASSIS, A.M.; ÁTILA, S.R.A.; MÓGOR, F. Extrato da alga Ascophyllum nodosum (L.) Le Jolis no desenvolvimento vegetativo e na produção do tomateiro. Amaz̧onian Journal of Agricultural and Environmental Sciences, v. 55, n. 4, p. 282-287, 2012.

KUMAR, G.; SAHOO, D. Effect of seaweed liquid extract on growth and yield of Triticum aestivum var. Pusa Gold. Journal of Applied Phycology, v. 23, p. 251255, 2011.

LEÃO, P.C.S.; SOARES, J.M.; RODRIGUES, B.L. Principais cultivares. In: SOARES, J.M.; LEAO, P.C.S. (Ed.). A vitivinicultura no Semiárido brasileiro. Brasília, DF: Embrapa Informação Tecnológica, 2009. cap. 5, p. 151-214.

MACKINNON, S.L.; HILTZ, D.; UGARTE, R.; CRAFT, C.A. Improved methods of analysis for betaines in Ascophyllum nodosum and its commercial seaweed extracts. Journal of Applied Phycology, v. 22, p. 489-494, 2010.

MARTYNENKO, A.; SHOTTON, K.; ASTATKIE, T.; PETRASH, G.; FOWLER, C.; NEILY, W.; CRITCHLEY, A. T. Thermal imaging of soybean response to drought stress: the effect of Ascophyllum nodosum seaweed extract. SpringerPlus, v. 5, n. 1, p. 1393, 2016.

PINILLOS, V.; CHIAMOLERA, F.M., ORTIZ, J.F., HUESO, J.J.; CUEVAS, J. Post-veraison regulated deficit irrigation in 'Crimson Seedless' table grape saves water and improves berry skin color. Agricultural Water Management, v. 165, p. 181-189, 2016

SABIR, A.; YAZAR, K.; SABIR, F.; KARA, Z.; YAZICI, M.A; GOKSU, N. Vine growth, yield, berry quality attributes and leaf nutrient content of grapevines as influenced by seaweed extract (Ascopbyllum nodosum) and nanosize fertilizer pulverizations. Scientia Horticulturae, v. 175, p. 1-8, 2014.
SHARMA, S.H.S.; LYONS, G.; McROBERTS, C.; McCALL, D.; CARMICHAEL, E.; ANDREWS, F.; SWAN, R.; McCORMACK, R.; MELLON, R. Biostimulant activity of brown seaweed species from Strangford Lough: compositional analyses of polysaccharides and bioassay of extracts using mung bean (Vigno mungo L.) and pak choi (Brassica rapa chinensis L.). Journal of Applied Phycology, v. 24, p. 10811091, 2012.

SILVA, C.C.; ARRAIS, I.G.; ALMEIDA, J.P.N.; DANTAS, L.L.G.R.; SILVA, F.S.O.; MENDONÇA, V. Extrato da alga Ascophyllum nodosum (L.) Le Jolis na produção de porta-enxertos de Anonna glabra L. Revista de Ciências Agrárias, v. 39, n. 2, p. 234-241, 2016.

SILVA, D.J.; LEÃO, P.C.S.; SANTOS, A.R.L.; SILVA, J.M.S; NASCIMENTO, L.A.; BRANDÃO, L.S. Efeito de bioestimulantes no desenvolvimento de mudas de videira. In: CONGRESSO BRASILEIRO DE FRUTICULTURA, 22, 2012, Bento Gonçalves, RS. Anais... Bento Gonçalves: SBF, 2012.

SOUZA, E.R.; AMARO, A.C.E.; SANTOS, L.S.; ONO, E.O.; RODRIGUES, J.D. Fenologia e trocas gasosas da videira cv. Sweet Sunshine em clima semiárido. Comunicata Scientiae, v. 7, n. 3, p. 319-333, 2016.

SPAEPEN, S.; VANDERLEYDEN, J.; OKON, Y. Plant growth promoting actions of rhizobacteria. Advances in Botanical Research, v. 51, p. 283-320, 2009.

SPANN, T.M.; LITTLE, H.A. Applications of a Commercial Extract of the Brown Seaweed Ascophyllum nodosum Increases Drought Tolerance in Containergrown 'Hamlin' Sweet Orange Nursery Trees. Hortscience, v. 46, n. 4, p. 577-582, 2011.

WALLY, O.S.D.; CRITCHLEY, A.T.; HILTZ, D.; CRAIGIE, J.S.; HAN, X.; ZAHARIA, L.I.; ABRAMS, S.R.; PRITHIVIRAJ, B. Regulation of Phytohormone Biosynthesis and Accumulation in Arabidopsis Following Treatment with Commercial Extract from the Marine Macroalga Ascophyllum nodosum. Journal of Plant Growth Regulation, v. 32, p. 324-339, 2013. 